OPEN

SUBJECT AREAS:

OPTICAL MATERIALS AND

STRUCTURES

LASERS

NANOPHOTONICS

APPLIED PHYSICS

Received

7 June 2012

Accepted

16 August 2012

Published

4 September 2012

Correspondence and requests for materials should be addressed to X.W.S. (EXWSun@ntu. edu.sg)

\title{
Strongly linearly polarized low threshold lasing of all organic photonic quasicrystals
}

\author{
D. Luo', Q. G. Du' , H. T. Dai ${ }^{2}$, H. V. Demir ${ }^{3,4}$, H. Z. Yang ${ }^{5}$, W. Ji ${ }^{5}$ \& X. W. Sun ${ }^{1,2}$
}

\begin{abstract}
'School of Electrical and Electronic Engineering, Nanyang Technological University, Nanyang Avenue, Singapore 639798, Singapore, ${ }^{2}$ Department of Applied Physics, College of Science, and Tianjin Key Laboratory of Low-Dimensional Functional Material Physics and Fabrication Technology, Tianjin University, Tianjin 300072, China, ${ }^{3}$ School of Electrical and Electronic Engineering, and School of Physical and Mathematical Sciences, Nanyang Technological University, Nanyang Avenue, Singapore 639798, Singapore, ${ }^{4}$ Department of Electrical and Electronics Engineering, Department of Physics, and UNAM - National Nanotechnology Research Center, Bilkent University, Bilkent, Ankara, 06800 Turkey, ${ }^{5}$ Department of Physics, National University of Singapore, 2 Science Drive 3, Singapore 117542, Singapore.
\end{abstract}

Lasing is obtained from a two-dimensional (2D) Penrose photonic quasicrystal made of a low index contrast material of holographic polymer dispersed liquid crystals (H-PDLCs) that enables a substantial reduction in the optical pumping threshold. This lasing architecture further allows for excellent linear polarization characteristics as well as wide directional dependence. The pumping threshold is fivefold lower than that obtained from the 2D defect-free photonic crystals fabricated under similar conditions. These properties make H-PDLC photonic quasicrystal promising for a new type of all organic miniature lasers.

hotonic quasicrystals, first observed by D. Shechtman in Al-Mn metallic alloys ${ }^{1}$, exhibit neither translation symmetry nor periodicity but possess a long-range order and orientational symmetry ${ }^{2}$. Similar to photonic crystals, there exist optical bandgaps in the photonic quasicrystals ${ }^{3-6}$. Photonic quasicrystals, fabricated in semiconductor materials, have been reported to generate unlimited lasing modes as a result of the arbitrary order of their rotational symmetry in contrast to the limited lasing modes in photonic crystals (PCs ${ }^{7-9}$.

Holographic polymer dispersed liquid crystals (H-PDLCs) have received substantial attention in fabricating PCs/photonic quasicrystals owing to their easy processing and tunable optoelectronic properties ${ }^{10-12}$. Despite the low index contrast excluding the formation of complete bandgaps, PCs made by low index contrast materials such as H-PDLCs can still create strongly coupled electromagnetic bands that yield low group velocity dispersion over a wide range of wave vectors, resulting in local field enhancement ${ }^{13}$. In fact, lasing for dye-doped 2D H-PDLC PCs have been reported ${ }^{14-16}$.

In this paper, we report for the first time the lasing of an all organic 2D H-PDLC Penrose photonic quasicrystal that achieves low pumping threshold and excellent linear polarization property as well as wide directional dependence, showing fundamental advantages over the lasing from typical 2D H-PDLC PC with high pumping threshold, weak polarization property, and narrow directional dependence. This work shows that lasing emission can be accomplished in 2D Penrose photonic quasicrystals H-PDLC of even very small index contrast materials.

\section{Results}

The 2D Penrose photonic quasicrystal was holographically fabricated through a five-beam interference generated by a specially designed prism. Figure 1(a) shows the schematic of the prism used (with a refractive index of 1.52), whose bottom plane is an equilateral pentagon with an equal side length of $3.2 \mathrm{~cm}$ and an equal internal angle of $108^{\circ}$. The side-bottom plane angle of the prism is $\Phi=60^{\circ}$. A collimated $\mathrm{Ar}^{+}$laser beam (at $514.5 \mathrm{~nm}$ ) impinging normally onto the prism (normal to the bottom plane) was used to generate five beams with wave vectors of $\boldsymbol{k}_{\mathbf{1}} \sim$ $\boldsymbol{k}_{5}$ (Figure 1(b)). The wave vectors can be written as

$$
\boldsymbol{k}_{\boldsymbol{m}}=k\left(-\cos \frac{2(m-1) \pi}{5} \sin \theta,-\sin \frac{2(m-1) \pi}{5} \sin \theta,-\cos \theta\right),
$$

where $m$ is an integer from 1 to $5, k=2 \pi n / \lambda$ is the unit wave vector, $\lambda$ is the optical writing wavelength in the air, and $n$ is the index of recording material. Here we have $\theta_{1} \sim \theta_{5}=\theta=25.3^{\circ}$. The interference pattern produced at 


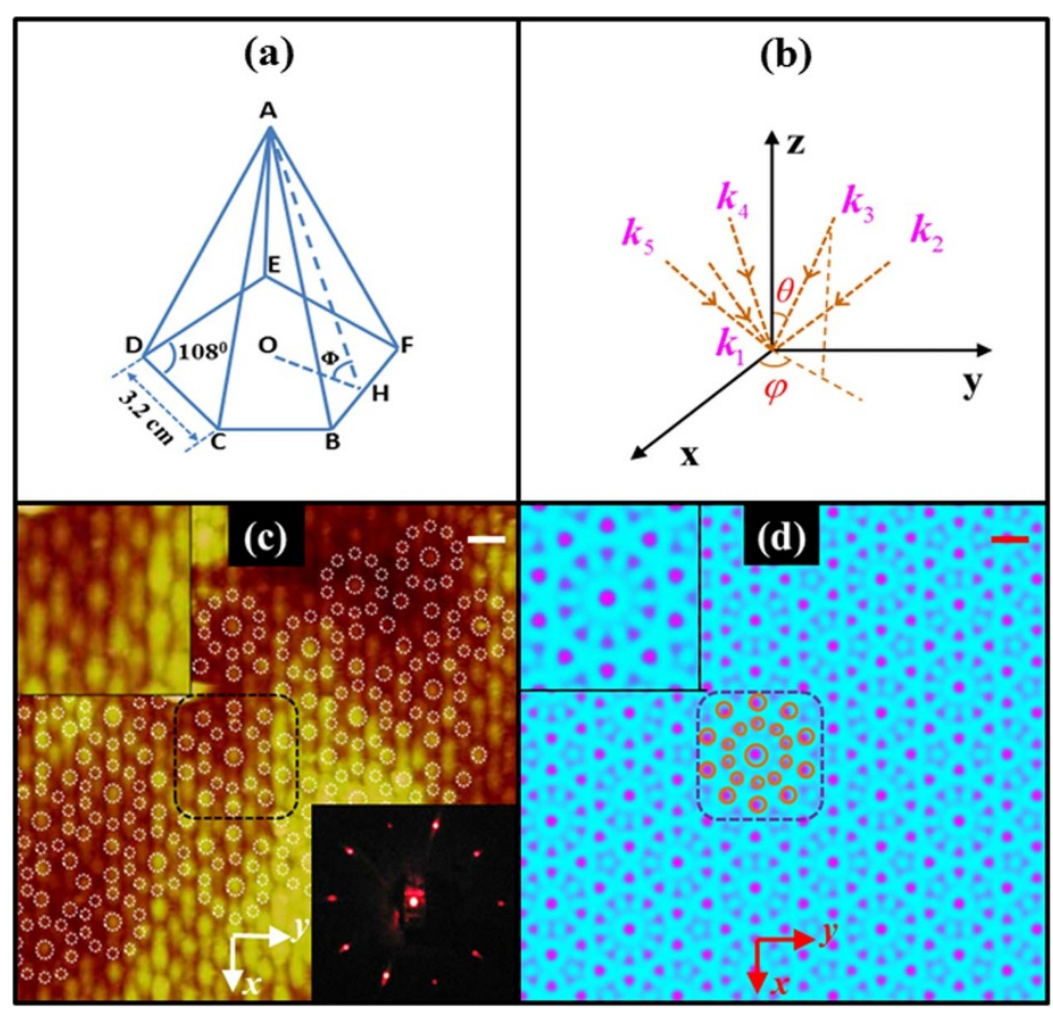

Figure 1 Five-beam interference and photonic quasicrystal structure. (a) Specially designed prism for five-beam generation. (b) Five-beam interference configuration, where the five beams with wave vectors of $\boldsymbol{k}_{\mathbf{1}} \sim \boldsymbol{k}_{\mathbf{5}}$ are generated through the refraction of five tilted side surfaces of the prism. $\theta$ represents the angle between the beam and vertical $z$ axis, and $\varphi$ represents the angle between the projection of wave vector on the $x-y$ plane and the $x$ axis. (c) AFM image showing the surface morphology of the 2D H-PDLC Penrose photonic quasicrystal. The light-colored and dark-colored regions represent polymer (marked by the dash circle) and LC, respectively. Scale bar: $1 \mu \mathrm{m}$. The inset shows the diffraction image of the 2D H-PDLC Penrose photonic quasicrystal, where 10 diffraction points confirm the five-fold symmetry structure of our sample. (d) Simulated intensity distribution of the five-beam interference pattern. The purple and blue areas represent high and low intensities, corresponding to polymer-rich (marked by the solid circle) and LC-rich regions, respectively. Scale bar: $1 \mu \mathrm{m}$.

the bottom surface of the prism in the overlapped area of five beams was recorded by a cell filled with LC/prepolymer mixture according to the polymerization induced phase separation ${ }^{15}$. As the five beams possess the same phase, the intensity distribution of the interference pattern is given by

$$
I(\boldsymbol{r})=\Re\left(\sum_{l, m=1}^{5} E_{l} \cdot E_{m} \exp \left[i\left(\boldsymbol{k}_{l}-\boldsymbol{k}_{\boldsymbol{m}}\right) \cdot \boldsymbol{r}\right]\right),
$$

where $l$ and $m$ are integers from 1 to $5, E$ is the electric field, $\boldsymbol{r}=(x, y$, $z$ ) is the position vector, and $\Re[\ldots]$ denotes the real part of the argument.

Figure 1(c) shows an atomic force microscopy (AFM) image of the surface morphology of the 2D H-PDLC sample, where the bright and dark areas represent polymer and LC, respectively. The diffraction image is depicted in the inset of Figure 1(c), confirming the existence of fivefold Penrose H-PDLC photonic quasicrystals ${ }^{12}$. For five beams with equal intensity, $E_{1} \sim E_{5}=E$, the simulated interference pattern in the $x-y$ plane is shown in Figure 1(d), where the red- and blue-colored regions represent high and low intensities, corresponding to the polymer-rich and LC-rich regions, respectively. In our simulation, we used $\lambda=514.5 \mathrm{~nm}$ and $n=1.533$ (calculated according to the ratio of the polymer and LC). The experimental result is in good agreement with the numerical simulation both in scale and morphology.

The schematic optical setup of our lasing experiment is presented in Figure 2(a). A linearly polarized pumping laser (with its electric field along the $x$ direction), focused by a cylinder lens, was incident on the surface of the sample in a shape of narrow line along the $y$ direction. Figure 2(b) shows the lasing spectra along the $y$ direction measured under different pumping energies. Multi-peaks (634.0 and
$637.0 \mathrm{~nm}$ ), whose linewidths are narrower than $1.6 \mathrm{~nm}$, were depicted on the profile of the lasing spectrum with a pumping energy up to $160 \mu \mathrm{J} /$ pulse. The peak intensity at the wavelength of $637 \mathrm{~nm}$ is illustrated as a function of the pumping energy in the inset of Figure 2(b), where the lasing threshold is $14 \mu \mathrm{J} /$ pulse.

Figure 2(c) shows the simulated transmittance, calculated by a commercial FDTD software package Lumerical, of Penrose quasicrystal along the $y$ direction in the range of 500-700 $\mathrm{nm}$, where the positions of 634.0 and $637.0 \mathrm{~nm}$ peaks are marked by a solid black line in a dash circle. These wavelengths located half way of the transmittance maxima and minima (such as the peaks of 634.0 and $637.0 \mathrm{~nm}$ ) are the positions for possible laser action because of the field enhancement of stimulated emission ${ }^{17}$. As the transmittance covers many transitions between the maxima and minima within $500-700 \mathrm{~nm}$, the lasing is expected to take place in 600$700 \mathrm{~nm}$, considering the overlapping range in $500-600 \mathrm{~nm}$ for both absorption and photoluminescence (PL) spectra of DCM (See Supplementary Information). The lasing from the QPC might be related to the so-called group velocity anomaly reflected from the transmission spectrum of the QPC. The group velocity anomaly refers to that the group velocity becomes smaller over a wide range of wave vectors. In our case of QPC, judging from the transmission spectrum, the lasing happens between the high and low transmittance, which is the same as what has been observed in 2D PC with small index contrast ${ }^{13}$. However, the group velocity anomaly of QPC has never been studied so far, more research needs to be done to verify our hypothesis. It is noticed that the threshold obtained here is fivefold lower than that achieved in the 2D H-PDLC PC with the hexagonal structure fabricated under similar conditions, where the threshold was about $70 \mu \mathrm{J} /$ pulse $^{15}$. 

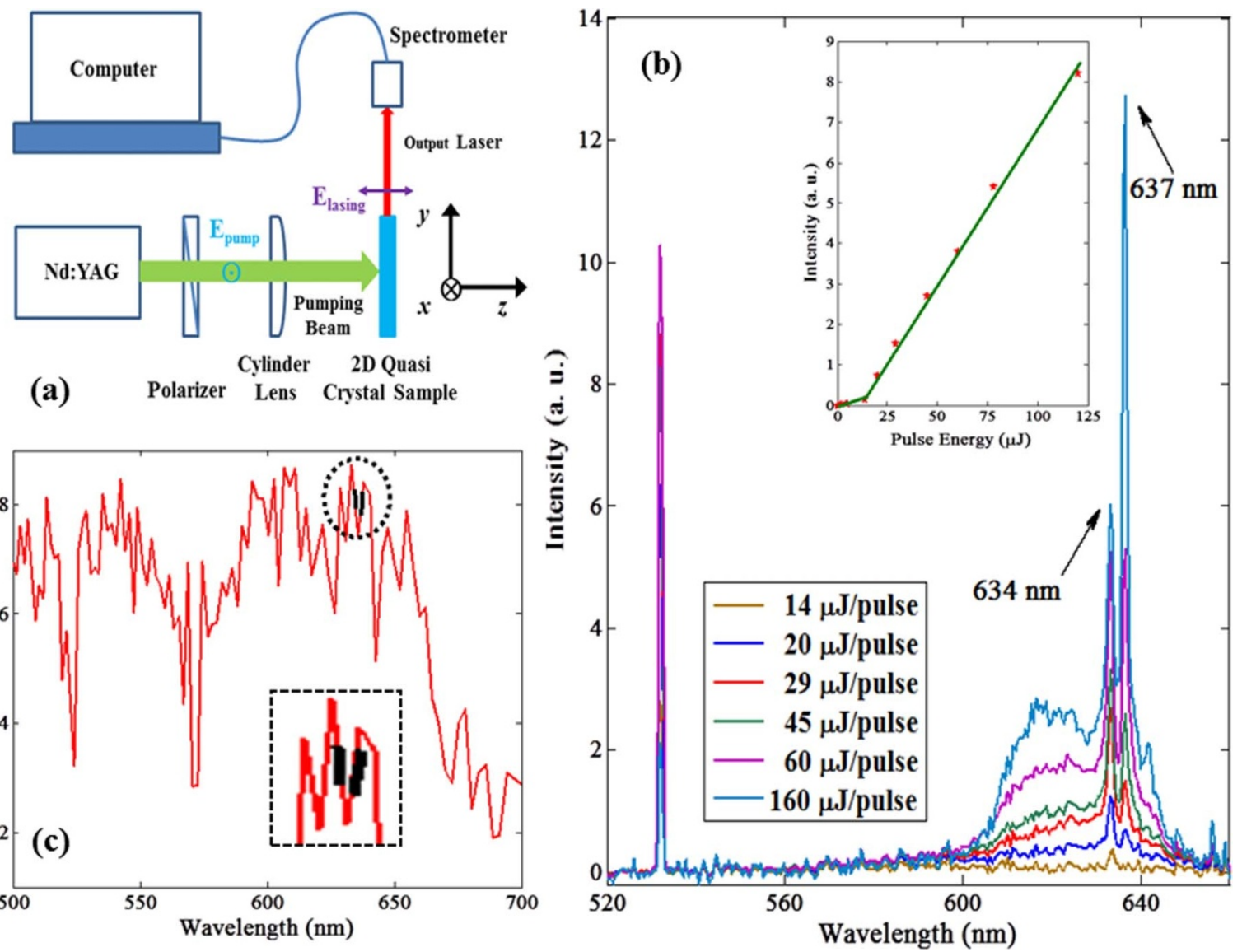

Figure 2 Lasing generation setup and lasing spectrum. (a) Schematic optical setup of the lasing experiment. A Q-switched frequency-doubled Nd:YAG pulsed laser operating at $532 \mathrm{~nm}$, with a pulse duration of $7 \mathrm{~ns}$ and a repetition rate of $10 \mathrm{~Hz}$, is used to pump the 2D dye-doped H-PDLC photonic quasicrystal sample. The linearly polarized pumping laser, focused by a cylinder lens, is incident on the surface of the sample in a shape of narrow line along the $y$ direction. The output lasing signals are collectted by a fiber coupled spectrometer. (b) Lasing spectra along the $y$ direction measured under different pumping energies. Multi-peaks of 634.0 and $637.0 \mathrm{~nm}$, whose linewidths are narrower than $1.6 \mathrm{~nm}$, are presented with the pumping energy up to $160 \mu \mathrm{J} /$ pulse. The inset depicts the dependences of the peak intensity on the pumping energy at the peak wavelength of $637.0 \mathrm{~nm}$. The threshold is 14 $\mu \mathrm{J} /$ pulse. (c) Simulated transmittance of Penrose quasicrystal along the $y$ direction. The dashed circles mark the peaks of 634.0 and $637.0 \mathrm{~nm}$. A blow up of the dashed circled spectrum is shown inset.

The lasing obtained from the 2D H-PDLC organic Penrose photonic quasicrystal exhibits an excellent linear polarization property, with the polarization along the $z$ direction (TM polarization). Figure 3(a) shows the lasing spectrum collected by adding a polarizer before the spectrometer at the pumping power of $92 \mu \mathrm{J} /$ pulse, where the peaks appear at 634.0 and $637.0 \mathrm{~nm}$. In Figure 3(a), we can see that the laser is linearly polarized along the surface normal direction. For $0^{\circ}$-positioned polarizer (TM polarization), the lasing intensity is reduced due to the absorption of the polarizer itself. With increasing angle of the polarizer, the lasing intensity decreases correspondingly, and finally at the angle of $90^{\circ}$ (TE polarization, with the electric field along the $x$ axis), the lasing spectrum almost completely disappears.

It is worth mentioning that this excellent linear polarization property of lasing obtained here in the 2D H-PDLC photonic quasicrystal was not achieved in the 2D H-PDLC PC lasing under similar experimental conditions ${ }^{16}$. Figures $3(\mathrm{~b})$ and $3(\mathrm{c})$ show the polarization property of the output lasing obtained from the 2D H-PDLC photonic quasicrystal and PC, respectively, where the lasing from the photonic quasicrystal exhibits excellent linear polarization property while the lasing from the PC does not.

\section{Discussion}

Figure 4 shows the cross-sectional field emission scanning electron microscopy (FESEM) images of the 2D H-PDLC PC (Fig. 4(a) and (b)) and 2D H-PDLC quasicrystal (Fig. 4(c) and (d)), respectively. The holes, which represent the space occupied by LC droplets, are marked by circles, while the period/quasi-period structures formed by polymer are marked by solid lines. In 2D H-PDLC PC (Fig. 4(e)), the LC droplets are primarily spherical and randomly positioned, resulting in an average index of LC $\left(n_{\text {ave }}=\left(n_{\mathrm{e}}+2 n_{\mathrm{o}}\right) / 3=1.5965\right)$ experienced both by TE and TM polarization. Therefore, both TE and TM polarization would have similar index contrasts and band structures, hence both TE and TM lasing can be observed even with similar intensity for 2D H-PDLC PC.

However, in 2D H-PDLC quasicrystal (Fig. 4(f)), our experiment process leads to a result of elliptical LC droplets with long axis parallel to $z$ axis, which results in a polarization dependent LC index; $n_{\mathrm{o}}=1.5216$ and $n_{\mathrm{e}}=1.7462$ for TE and TM polarization respectively. Because the index of polymer $n_{\mathrm{p}}(1.5220)$ is very close to $n_{\mathrm{o}}(1.5216)$, the TE polarization will experience very small index contrast and the 2D quasicrystal looks like an isotropic material. On the contrary, the TM polarization will experience a relatively larger index contrast $\left(n_{\mathrm{e}}\right.$ and $n_{\mathrm{p}}$ ). Hence the lasing from H-PDLC quasicrystal is polarized; there is only TM but no TE lasing.

It is worth mentioning that the TM polarization will experience an enhanced index contrast in 2D H-PDLC quasicrystal $\left(n_{\mathrm{e}}-n_{\mathrm{p}}=\right.$ $0.2242)$ compared to that in 2D H-PDLC PC $\left(n_{\text {ave }}-n_{\mathrm{p}}=0.0745\right)$, leading to a stronger feedback mechanism and hence lower lasing threshold in quasicrystal case. The higher-order rotation symmetry 

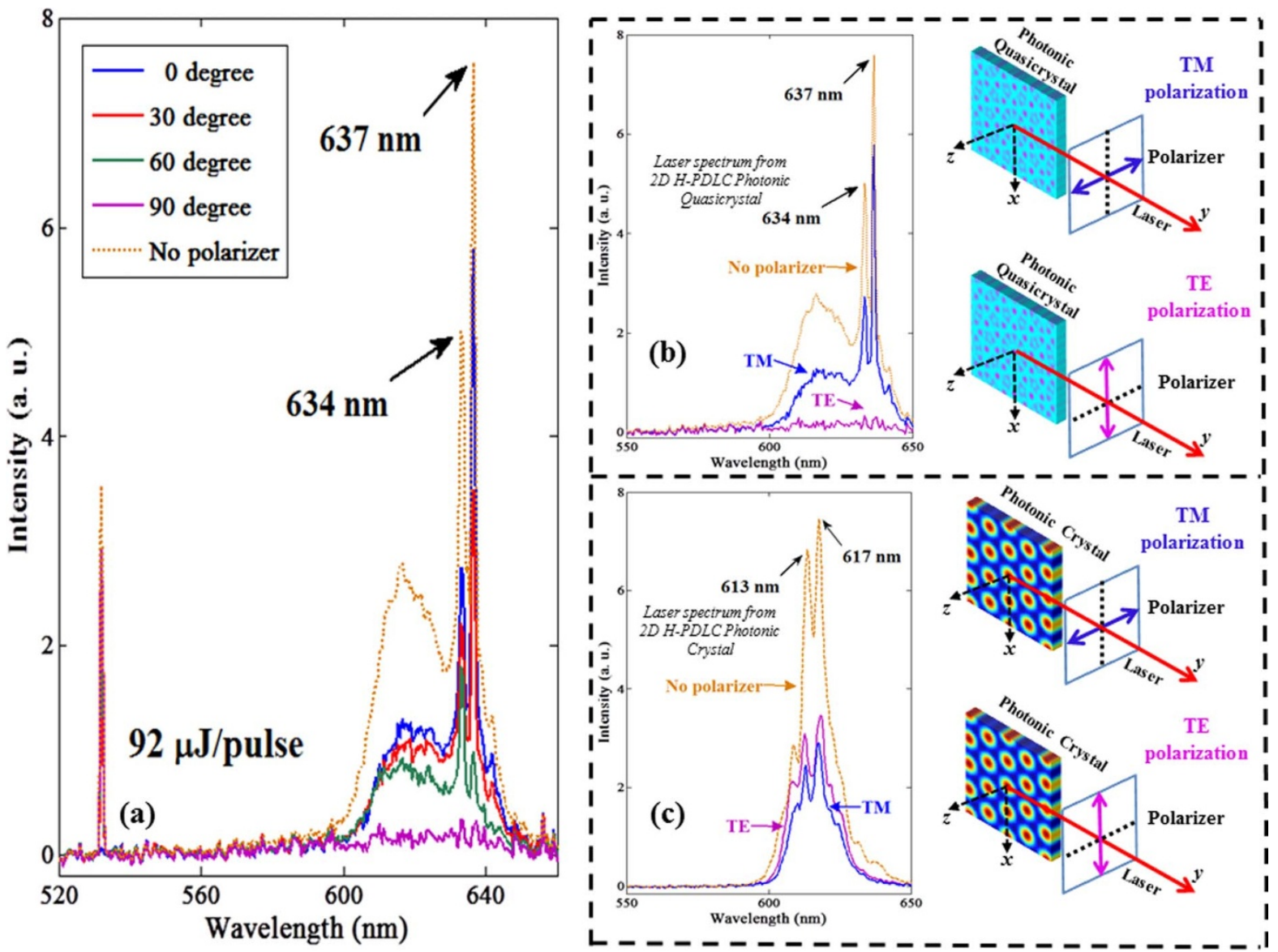

Figure 3 Linearly polarized lasing. (a) Lasing spectra under different polarization conditions. Dashed line represents the lasing spectrum obtained without a polarizer, and solid lines represent the lasing spectra obtained with the rotating polarizer. Specifically, $0^{\circ}$ and $90^{\circ}$ refer to the case that the direction of the polarizer is parallel to the $z$ and $x$ axis, respectively, and $30^{\circ} / 60^{\circ}$ means the direction of the polarizer is tilted toward the $z$ axis by $30^{\circ} / 60^{\circ}$. (b) Laser spectra obtained with no polarizer, $\mathrm{TM}\left(0^{\circ}\right)$ polarization, and TE $\left(90^{\circ}\right)$ polarization from the $2 \mathrm{D}$ H-PDLC photonic quasicrystal, comparing with (c) the laser spectra obtained with no polarizer, TM polarization, and TE polarization from the 2D H-PDLC photonic crystal (for which the experimental detail can be found from Ref. 16).

in quasicrystal is another possible reason for the lower pumping threshold in quasicrystal than photonic crystal.

The lasing from our 2D H-PDLC Penrose photonic quasicrystal was also studied in different directions at a pumping power level of $160 \mu \mathrm{J} /$ pulse (Figure 5). Figure 5 (a) shows the pseudo-Jones zone of the Penrose quasicrystal in reciprocal space, which is corresponding to the first Brillouin zone in the photonic crystal case ${ }^{18}$. The directions of $\Gamma \mathrm{X}, \Gamma \mathrm{N}$, and $\Gamma \mathrm{X}$ are overlapped with the $0^{\circ}, 18^{\circ}$, and $36^{\circ}$ directions marked by red, blue and purple in Figure 5(b) respectively. We can see from Figure 5(c) that a lasing red-shift was observed while the direction was changed from angle $0^{\circ}$ to $18^{\circ}$ with respect to the $y$ axis, where the main peak shifted from 634.0/637.0 to $668.5 \mathrm{~nm}$. At the angle of $0^{\circ}$, the lasing peaks were only at $634.0 /$ $637.0 \mathrm{~nm}$. At the angle of $9^{\circ}$, the lasing peaks of $634.0 / 637.0 \mathrm{~nm}$ were largely supressed and the peak of $668.5 \mathrm{~nm}$ started to appear (while a peak of $625.5 \mathrm{~nm}$ also emerged). At the angle of $18^{\circ}$, the lasing peaks of $634.0 / 637.0 \mathrm{~nm}$ were still suppressed but the peak of $668.5 \mathrm{~nm}$ dominated the spectrum (while the peak of $625.5 \mathrm{~nm}$ was kept at a low intensity level). We can see that the different lasing modes have their preferred lasing directions, where the symmetry directions of $\Gamma \mathrm{X}$ and $\Gamma \mathrm{N}$ are favored. A reverse lasing blue-shift was then observed while the direction was changed from angle $18^{\circ}$ to $36^{\circ}$ with respect to the $y$ axis, where the main peak shifted from 668.5 back to 634.0/ $637.0 \mathrm{~nm}$. Since the Penrose photonic quasicrystal possesses the rotational symmetry, with a rotational angle of $36^{\circ}$, and the axial symmetry, the experienced structure is actually the same for light
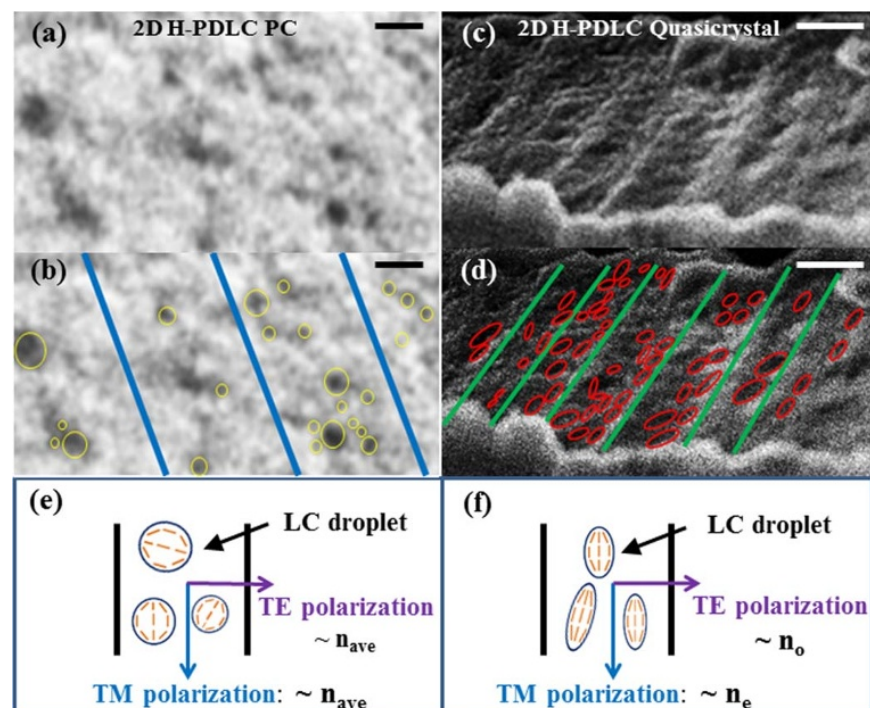

(f)

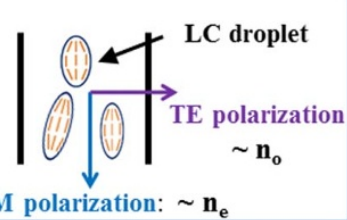

Figure $4 \mid$ Liquid crystal droplets shape. Cross-sectional FESEM images of (a), (b) 2D H-PDLC PC (Scalar bar: $200 \mathrm{~nm}$ ) and (c), (d) 2D H-PDLC quasicrystal (Scalar bar: $1 \mu \mathrm{m}$ ). The holes left by LC droplets are marked by circles/ellipses, while period/quasi-period structures formed by polymer are marked by solid lines. Illustration of the LC droplets in (e) 2D H-PDLC $\mathrm{PC}$ and (f) 2D H-PDLC quasicrystal with their corresponding refractive indices experienced. 


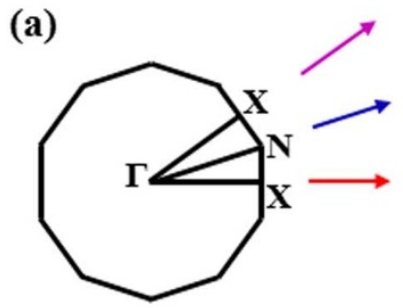

(c)

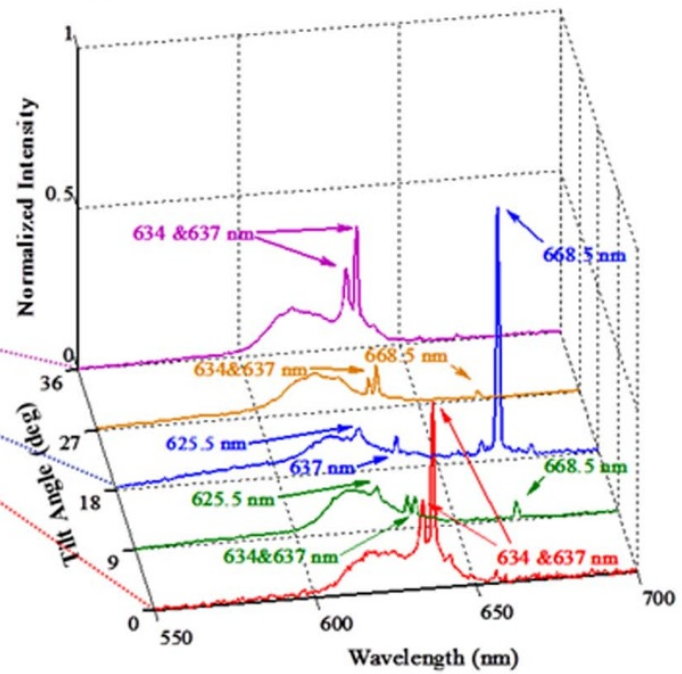

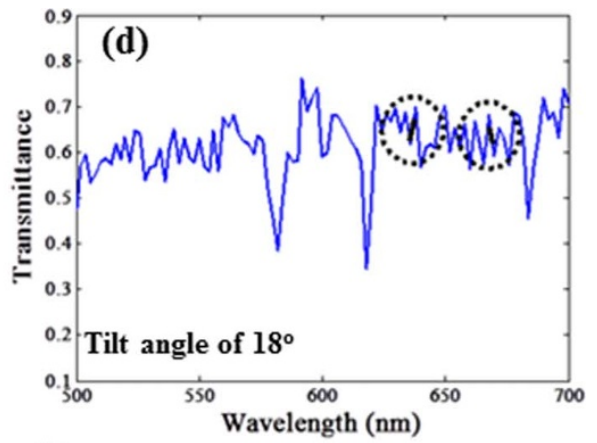

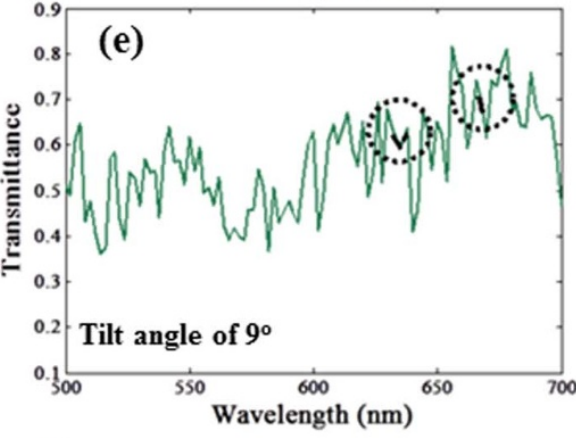

(b)
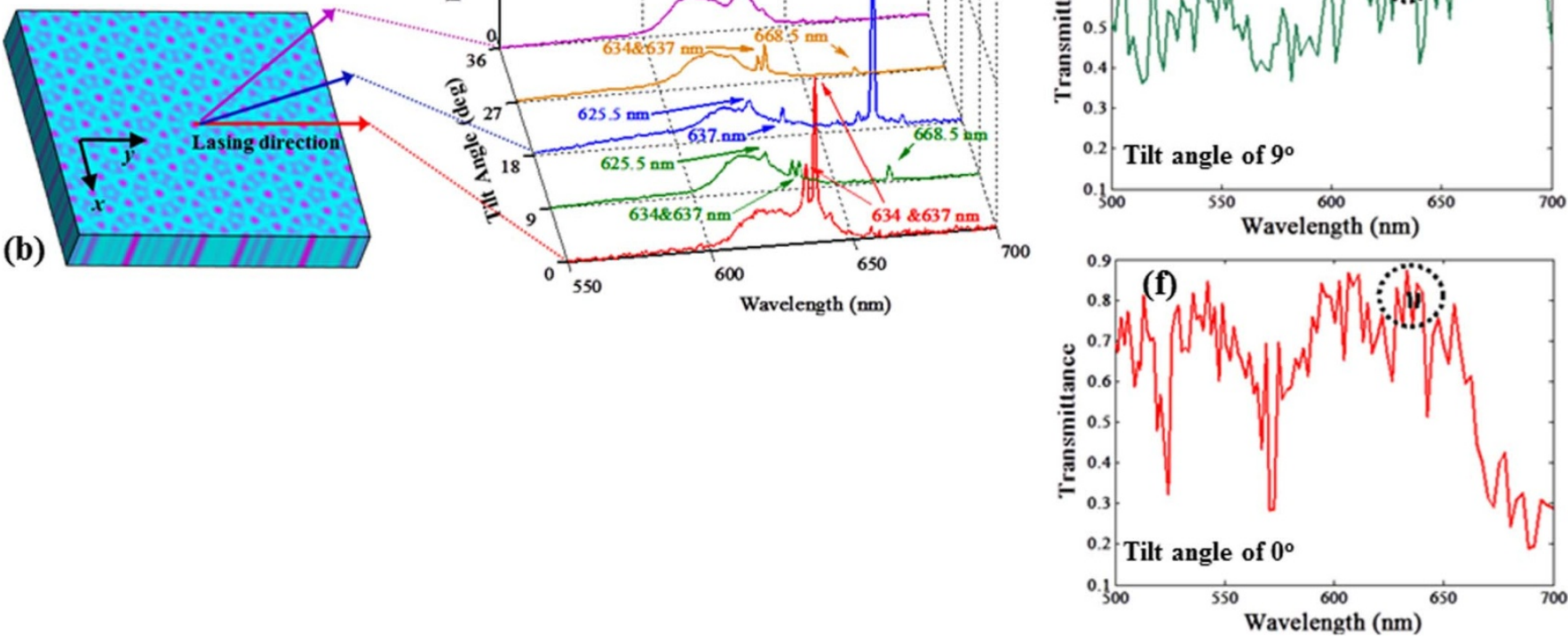

Figure 5 Lasing along different directions. (a) Pseudo-Jones zone in reciprocal space with symmetry point. (b) Schematic of the lasing generated along different directions within the 2D Penrose photonic quasicrystal. (c) Spectra of the lasing measured at $160 \mu \mathrm{J} /$ pulse with the tilt angle changing from $0^{\circ}$ to $36^{\circ}$. A red-shift of the lasing peak wavelength is observed initially, which is then followed by a blue-shift. (d)-(f) Calculated transmittance spectra along the directions with the tilt angle of $0^{\circ}, 9^{\circ}$, and $18^{\circ}$, respectively. Corresponding wavelengths of the laser peaks $(634.0 / 637.0 / 668.5 \mathrm{~nm})$ are marked by the solid line with a dashed circle.

oscillating at the direction pairs of $0^{\circ} / 36^{\circ}$ and $9^{\circ} / 27^{\circ}$, respectively. The same structures lead to the same lasing oscillation conditions, causing lasing spectrum to red-shift initially and then to blue-shift back from the halfway on. The calculated transmittance spectra along the tilt angles of $0^{\circ}, 9^{\circ}$, and $18^{\circ}$ are also shown in Figure $5(\mathrm{~d})$ to (f) respectively, where the corresponding wavelengths of the laser peaks are marked by dashed circles. The transmittance spectrum along $0^{\circ}$ is shown here again for comparison purposes.

In conclusion, we demonstrated low threshold lasing dye-doped 2D Penrose photonic quasicrystals fabricated by H-PDLC, by means of which an excellent linear polarization (TM polarization only) property was also observed. A wide directional dependence of the lasing was further demonstrated, where the lasing wavelength was tunable by changing the lasing generation directions in the photonic quasicrystal. These properties make H-PDLC photonic quasicrystal promising for a new type of all organic mirrorless miniature lasers, opening new possibilities for organic laser architectures.

\section{Methods}

In our experiment, the liquid crystal (LC)/prepolymer mixture syrup used to fabricate the dye-doped 2D PC with a hexagonal lattice structure consisted of $63.76 \mathrm{wt} \%$ monomer, trimethylolpropane triacrylate (TMPTA), $7.05 \mathrm{wt} \%$ cross-linking monomer, $N$-vinylpyrrollidone (NVP), $0.49 \mathrm{wt} \%$ photoinitiator, Rose Bengal (RB), 0.98 wt\% coinitiator, $N$-phenylglycine (NPG), 9.30 wt\% surfactant, octanoic acid (OA), and $1.18 \mathrm{wt} \%$ lasing dye, 4-dicyanomethylene-2-methyl-6-p-dimethylaminostyryl$4 \mathrm{H}$-pyran (DCM), all from Sigma-Aldrich, and $17.24 \mathrm{wt} \%$ liquid crystal, E7 $\left(n_{\mathrm{o}}=\right.$ 1.5216 and $n_{\mathrm{e}}=1.7462$ ), from Merck. The mixture was sandwiched in a cell, which was formed by two pieces of indium tin oxide (ITO) coated glass.

In the quasicrystal fabrication, the exposure intensity of each beam and exposure time were $\sim 5 \mathrm{~mW} / \mathrm{cm}^{2}$ and $120 \mathrm{~s}$, respectively. The recording area of the $2 \mathrm{D}$ Penrose photonic quasicrystal sample was about $5 \times 5 \mathrm{~mm}^{2}$ in the $x-y$ plane with a film thickness of $4 \mu \mathrm{m}$ (in the $z$ direction). The AFM image of the sample was obtained after LC was removed from ethanol (after over 12 hours soaking). For the lasing generation, a Q-switched frequency-doubled Nd:yttrium-aluminum-garnet (Nd:YAG) pulsed laser operating at $532 \mathrm{~nm}$, with a pulse duration of $7 \mathrm{~ns}$ and a repetition rate of $10 \mathrm{~Hz}$, was used to pump the 2D dye-doped H-PDLC photonic quasicrystal sample. The output lasing signal was collected by a fiber coupled spectrometer connected to a computer with a resolution of $0.6 \mathrm{~nm}$ at room temperature.

1. Shechtman, D., Blech, I., Gratias, D. \& Cahn, J. W. Metallic phase with long-range orientational order and no translational symmetry. Phys. Rev. Lett. 53, 1951-1953 (1984).

2. Rotenberg, E., Theis, W., Horn, K. \& Gille, P. Quasicrystalline valence bands in decagonal AlNiCo. Nature 406, 602-605 (2000).

3. Chan, Y. S., Chan, C. T. \& Liu, Z. Y. Photonic band gaps in two dimensional photonic quasicrystals. Phys. Rev. Lett. 80, 956-959 (1998).

4. Zoorob, M. E., Charlton, M. D. B., Parker, G. J., Baumberg, J. J. \& Netti, M. C. Complete photonic bandgaps in 12-fold symmetric quasicrystals. Nature 404, 740-743 (2000).

5. Man, W., Megens, M., Steinhardt, P. J. \& Chaikin, P. M. Experimental measurement of the photonic properties of icosahedral quasicrystals. Nature 436, 993-996 (2005). 
6. Freedman, B., Bartal, G., Segev, M., Lifshitz, R., Christodoulides, D. N. \& Fleischer, J. W. Wave and defect dynamics in nonlinear photonic quasicrystals. Nature $\mathbf{4 4 0}$ 1166-1169 (2006).

7. Mahler, L., Tredicucci, A., Beltram, F., Walther, C., Faist, J., Beere, H. E., Ritchie, D. A. \& Wiersma, D. S. Quasi-periodic distributed feedback laser. Nat. Photon. 4 165-169 (2010).

8. Kok, M. H., Lu, W., Tam, W. Y. \& Wong, G. K. L. Lasing from dye-doped icosahedral quasicrystals in dichromate gelatin emulsions. Opt. Express 17, 7275-7284 (2009).

9. Notomi, M., Suzuki, H., Tamamura, T. \& Edagawa, K. Lasing action due to the two-dimensional quasiperiodicity of photonic quasicrystals with a Penrose lattice. Phys. Rev. Lett. 92, 123906 (2004).

10. Bunning, T. J., Natarajan, L. V., Tondiglia, V. P. \& Sutherland, R. L. Holographic polymer-dispersed liquid crystals (H-PDLCs). Annu. Rev. Mater. Sci. 30, 83 (2000)

11. Sutherland, R. L., Tondiglia, V. P., Natarajan, L. V., Chandra, S., Tomlin, D. \& Bunning, T. J. Switchable orthorhombic F photonic crystals formed by holographic polymerization-induced phase separation of liquid crystal. Opt. Express 10, 1074-1082 (2002).

12. Gorkhali, S. P., Qi, J. \& Crawford, G. P. Switchable quasi-crystal structures with five-, seven-, and ninefold symmetries. J. Opt. Soc. Am. B 23, 149-158 (2006).

13. Sakoda, K. Enhanced light amplification due to group-velocity anomaly peculiar to two- and three-dimensional photonic crystals. Opt. Express 4, 167-176 (1999).

14. Jakubiak, R., Tondiglia, V. P., Natarajan, L. V., Sutherland, R. L., Lloyd, P., Bunning, T. J. \& Vaia, R. A. Dynamic lasing from all-organic two-dimensional photonic crystals. Adv. Mat. 17, 2807-2811 (2005).

15. Luo, D., Sun, X. W., Dai, H. T., Demir, H. V., Yang, H. Z. \& Ji, W. Two-directional lasing from a dye-doped two-dimensional hexagonal photonic crystal made of holographic polymer-dispersed liquid crystals. Appl. Phys. Lett. 95, 151115 (2009).

16. Luo, D., Sun, X. W., Dai, H. T., Demir, H. V., Yang, H. Z. \& Ji, W. Electrically tunable lasing from a dye-doped two-dimensional hexagonal photonic crystal made of holographic polymer-dispersed liquid crystals. Appl. Phys. Lett. 97, $081101(2010)$
17. Negro, L. D., Oton, C. J., Gaburro, Z., Pavesi, L., Johnson, P., Lagendijk, A., Righini, R., Colocci, M. \& Wiersma, D. S. Light transport through the band-edge states of Fibonacci quasicrystals. Phys. Rev. Lett. 90, 055501 (2003).

18. Kaliteevski, M. A., Brand, S., Abram, R. A., Krauss, T. F., De La Rue, R. M. \& Millar, P. Two-dimensional Penrose-tiled photonic quasicrystals: diffraction of light and fractal density of modes. J. Mod. Optic. 47, 1771-1778 (2000).

\section{Acknowledgements}

This work is supported by the Singapore A*STAR (Grant No. 0921010057), National Research Foundation (NRF-CRP-6-2010-02 and NRF-RF-2009-09), National Natural Science Foundation of China (NSFC) (Project Nos. 61006037, 61177014 and 61076015), and Tianjin Natural Science foundation (Project Nos. 11JCZDJC21900 and 11JCYDJC25800)

\section{Author contributions}

D. Luo and X. W. Sun conceived the experiments. D. Luo prepared the samples and conducted the lasing experiment. Q. G. Du performed the numerical simulations. D. Luo, H. T. Dai, H. V. Demir and X. W. Sun discussed and contributed to the final version of manuscript. H. Z. Yang and W. Ji assisted with lasing measurement. X. W. Sun supervised the whole project.

\section{Additional information}

Supplementary information accompanies this paper at http://www.nature.com/ scientificreports

Competing financial interests: The authors declare no competing financial interests.

License: This work is licensed under a Creative Commons

Attribution-NonCommercial-ShareAlike 3.0 Unported License. To view a copy of this license, visit http://creativecommons.org/licenses/by-nc-sa/3.0/

How to cite this article: Luo, D. et al. Strongly linearly polarized low threshold lasing of all organic photonic quasicrystals. Sci. Rep. 2, 627; DOI:10.1038/srep00627 (2012). 